Published in final edited form as:

Mol Carcinog. 2014 February ; 53(0 1): E130-E139. doi:10.1002/mc.22054.

\title{
Slug ${ }^{\mathrm{1}}$ Expression Inhibits Calcitriol-Mediated Sensitivity to Radiation in Colorectal Cancer
}

\author{
Victoria J. Findlay ${ }^{1,}$, R. Eric Moretz ${ }^{2}$, Cindy Wang ${ }^{2}$, Silvia G. Vaena ${ }^{2}$, Savannah G. \\ Bandurraga $^{1}$, Michael Ashenafi ${ }^{3}$, David T. Marshall ${ }^{3}$, Dennis K. Watson ${ }^{1,4}$, and E. Ramsay \\ Camp ${ }^{2,5}$ \\ ${ }^{1}$ Department of Pathology and Laboratory Medicine, Medical University of South Carolina, \\ Charleston, South Carolina \\ ${ }^{2}$ Department of Surgery, Medical University of South Carolina, Charleston, South Carolina \\ ${ }^{3}$ Department of Radiation Oncology, Medical University of South Carolina, Charleston, South \\ Carolina \\ ${ }^{4}$ Department of Biochemistry and Molecular Biology, Medical University of South Carolina, \\ Charleston, South Carolina \\ ${ }^{5}$ Ralph H. Johnson VA Medical Center, Charleston, South Carolina
}

\begin{abstract}
Recently, a reciprocal relationship between calcitriol and epithelial-to-mesenchymal transition has been described. Therefore, we hypothesized that calcitriol (1a,25-dihydroxyvitamin $\mathrm{D}_{3}$ ) would enhance radiation sensitivity in colorectal cancer regulated by epithelial mesenchymal transition. Vitamin-D receptor, E-cadherin and vimentin protein as well as E-cadherin, Snail and Slug mRNA levels were assessed in a panel of human colorectal cancer cell lines at baseline and in response calcitriol. We defined cell lines as calcitriol sensitive based on demonstrating an enhanced epithelial phenotype with increased E-cadherin, reduced vimentin and decreased expression of Snail and Slug as well as decreased cellular migration in response to calcitriol. In calcitriol sensitive cells, including DLD-1 and HCT116, $24 \mathrm{~h}$ calcitriol pre-treatment enhanced the radiation sensitivity by $2.3-$ and 2.6 -fold, respectively, at 4 Gy $(P<0.05)$. In contrast, SW620 cells with high baseline mesenchymal features including high Slug and vimentin expression with low Ecadherin expression demonstrated no significant radiation sensitizing response to calcitriol treatment. Similarly, transfection of Slug in the calcitriol sensitive colon cancer cell lines, DLD-1 and HCT 116, completely inhibited the radiation sensitizing effect of calcitriol. Collectively, we demonstrate that calcitriol can enhance the therapeutic effects of radiation in colon cancer cells and Slug expression mitigates this observed effect potentially representing an effective biomarker for calcitriol therapy.
\end{abstract}

\section{Keywords}

vitamin D; radiation; colorectal; cancer; EMT; Slug

\footnotetext{
(C) 2013 Wiley Periodicals, Inc.

*Correspondence to: Department of Pathology and Laboratory Medicine, Medical University of South Carolina, 39 Sabin Street, WRB, RS-310, Charleston, SC 29425.
} 


\section{INTRODUCTION}

Neoadjuvant chemoradiation (CMT) has become standard of care in locally advanced rectal cancer (LARC) patients [1]. However, tumor response is observed in only approximately $60 \%$ of the cases and only $20 \%$ have a complete response [1,2]. With the inconsistent response to CMT, novel therapeutic strategies are needed to improve results in this devastating disease.

The active metabolite of Vitamin $\mathrm{D}$, otherwise known as 1a,25-dihydroxyvitamin $\mathrm{D}_{3}$ (calcitriol) as well as Vitamin D analogues have demonstrated therapeutic potential as a single agent and in combination with chemotherapeutic agents in multiple Phase I clinical trials primarily focusing on prostate cancer patients [3,4]. Pre-clinical data supporting the use of calcitriol to enhance chemotherapy has appeared promising across a variety of malignancies including prostate, squamous cell carcinoma, and pancreatic cancer [5-7]. Similarly, calcitriol has demonstrated the ability to sensitize prostate cancer cells to radiation [8]. In addition, colon cancer cells treated with calcitriol demonstrate growth inhibition, but the ability of calcitriol to enhance therapeutic strategies in colon cancer is largely unexplored [9-11].

Recently, a reciprocal relationship between calcitriol and epithelial-to-mesenchymal transition (EMT) has been demonstrated [12-14]. Vitamin D receptor (VDR) activation by treatment with calcitriol has demonstrated the ability to inhibit cell growth associated with increased E-cadherin expression, suggesting a component of VDR's anti-neoplastic effect may occur by inhibiting EMT [12]. EMT-associated phenotypes, including enhanced motility and invasion, have been well characterized in cancer cells $[15,16]$. Recently, transcriptional mediators of EMT such as SNAII (Snail) and SNAI2 (Slug) have also been associated with enhanced cellular survival $[17,18]$. For instance, Snail expression in MadinDarby canine kidney cells attenuated cell death in response to serum starvation and TNF-a treatment. The anti-apoptotic response as a result of Snail expression was associated with activation of both MAPK and PI3K pathways [17]. Similarly, colon cancer cells resistant to oxaliplatin chemotherapy-induced apoptosis had alterations consistent with EMT including loss of cellular polarity, a spindle shape, increased motility and loss of membrane-bound Ecadherin expression [19]. Therefore, the ability of calcitriol to inhibit EMT may be a critical therapeutic mechanism by which calcitriol improves anti-neoplastic therapeutic strategies. In contrast, Snail and Slug can repress activity of the VDR promoter and inhibit VDR protein expression $[13,14,20]$. The reciprocal relationship between calcitriol and EMT led us to hypothesize that the ability of calcitriol to sensitize colorectal cancer cells to radiation will be regulated by critical EMT mediators.

\section{MATERIALS AND METHODS}

\section{Cell Lines and Culture Conditions}

Human colon cancer cell lines (DLD1, HCT116, HT29, SW480, SW620,) were cultured according to American Type Culture Collection recommendations and maintained at $37^{\circ} \mathrm{C}$ with 5\% $\mathrm{CO}_{2}$. Mycoplasma-negative cultures were ensured by PCR testing prior to the investigations (Stratagene, La Jolla, CA). Cells were monitored throughout the course of these studies and demonstrated consistent morphology and doubling time.

\section{Reagents}

Antibodies used for Western Blot analyses were as follows: mouse anti-E-cadherin antibody (BD Bioscience, San Jose, CA, 610181, 1:5000 dilution), anti-Vitamin D receptor (abcam, ab8756, 1:100 dilution), rabbit anti-vimentin (Cell Signaling Technology, Inc, Danvers, 
MA, cs3932, 1:2000 dilution), rabbit anti-GAPDH (abcam, Ab9485, 1:5000 dilution), and rabbit anti-actin (Sigma-Aldrich, St. Louis, MO, A2066, 1:2000 dilution). Goat anti-rabbit (1:5000 dilution) and horse anti-mouse (1:2000 dilution) horseradish peroxidase-conjugated antibodies were acquired from Cell Signaling Technology, Inc. (7074S, 7076S). Rabbit antirat (1:1000 dilution) horseradish peroxidase-conjugated secondary antibody was acquired from abcam (ab6734). Alexa Fluor 488-conjugated goat anti-mouse IgG (1:1000 dilution) was from Molecular Probes, Inc. (Life Technologies, Grand Island, NY, A-11001).

\section{Generation of Stable Cell Lines}

Slug was amplified from genomic DNA using Slug-specific primers using previously validated sequences [21]. The Snail pCMV-Tag2B vector was a generous gift from MienChie Hung (University of Texas, M.D. Anderson Cancer Center). For the generation of DLD-1 and HCT116 cells overexpressing Slug (Slug DLD-1), Snail (Snail DLD-1) or empty (empty DLD-1) pCMV-3Tag-1 vector were transfected using Lipofectamine 2000 according to the manufacturer's recommendations (Invitrogen, Carlsbad, CA, 11668-027). Stable transfected pools of cells were selected in medium containing G418 $(400 \mu \mathrm{g} / \mathrm{mL}$; Invitrogen). Gene expression was confirmed by qRT-PCR.

\section{Reverse Transcription-PCR (qRT-PCR)}

Total RNA from cultured cells was extracted using the RNeasy Plus Mini kit (Qiagen, Valencia, CA). Total RNA $(1 \mu \mathrm{g})$ was reverse transcribed in a $20 \mu \mathrm{L}$ reaction using iScript (Bio-Rad, Hercules, CA). qRT-PCR was performed with $5 \mu \mathrm{L}$ of a $1 / 20$ dilution of reversetranscribed cDNA for the cell line samples using the UPL mono-color probes in the Roche Light Cycler 480 machine (Roche Diagnostics). The cycling conditions for all genes included pre-incubation at $95^{\circ} \mathrm{C}$ for $10 \mathrm{~min}$ followed by 55 cycles for denaturation at $95^{\circ} \mathrm{C}$ for $15 \mathrm{~s}$ and amplification/extension at $60^{\circ} \mathrm{C}$ for $30 \mathrm{~s}$; after cycle completion, cooling was held for $30 \mathrm{~s}$ at $40^{\circ} \mathrm{C}$. Triplicate reactions were run for each cDNA sample. The relative expression of each gene was quantified on the basis of $\mathrm{Ct}$ value measured against an internal standard curve for each specific set of primers using the software provided by the instrument manufacturer (Roche Diagnostics). These data were normalized to glyceraldehyde-3phosphate dehydrogenase (GAPDH) and confirmed with biologic replicate samples. Sequences for gene-specific primers and probe numbers for Slug, E-cadherin and GAPDH are published [22]. Sequences for Snail are forward $5^{\prime}$-gctgcaggactctaatccaga- $3^{\prime}$ and reverse $5^{\prime}$-atctccggaggtgggatg- $3^{\prime}$ with UPL probe \#11, vimentin are forward $5^{\prime}$ aaagtgtggctgccaagaac- $3^{\prime}$ and reverse $5^{\prime}$-agcctcagagaggtcagcaa- $3^{\prime}$ with UPL probe \#16 and VDR are forward $5^{\prime}$-cttctctggggactcctcct- $3^{\prime}$ and reverse $5^{\prime}$-tggacgagtccatcatgtct- $3^{\prime}$ with UPL probe \#15.

\section{Western Blot Analysis}

Cells were suspended in radioimmunoprecipitation assay (RIPA) protein lysis buffer $(\mathrm{pH}$ 7.4), containing $20 \mathrm{mM}$ sodium phosphate, $150 \mathrm{mM}$ sodium chloride, $1 \%$ Triton $\mathrm{X}-100,5$ mM EDTA, $5 \mathrm{mM}$ phenylmethylsulfonyl fluoride, $1 \%$ aprotinin, $1 \mu \mathrm{g} / \mathrm{mL}$ leupeptin, and $500 \mu \mathrm{M} \mathrm{Na}_{3} \mathrm{VO}_{4}$. Protein concentration was quantified using Bio-Rad protein assay (BioRad, 500-0111). Twenty micrograms of total protein was resolved with SDS-PAGE (10\% polyacrylamide gel), and transferred to a polyvinylidene difluoride membrane.

Immunoblotting was performed with SuperSignal WestPico chemiluminescence substrate (Thermo Scientific, Logan, UT, 34080). Blots were probed with commercially available antibodies as outlined above. All membranes were stripped and reprobed with GAPDH antibody for loading control. 


\section{Immunofluorescence Analysis}

Cells were seeded as monolayers onto sterile, confocal glass coverslips (35 mm; MatTek Corp., Ashland, MA), coated with $5 \mu \mathrm{g} / \mathrm{mL}$ fibronectin in culture dishes and allowed to attach overnight. Following $48 \mathrm{~h}$ of treatment with either $1 \mu \mathrm{M}$ calcitriol (Sigma, D1530) or control, cells were then fixed for 10 min with $3.7 \%$ formaldehyde, washed three times with $1 \times$ phosphate-buffered saline (PBS), permeabilized with $0.1 \%$ Triton X-100, and blocked in $2 \%$ bovine serum albumin (BSA) in $1 \times \mathrm{PBS}$ for $1 \mathrm{~h}$ at room temperature. E-cadherin primary antibody (Santa Cruz Biotechnology, sc-21791) was used overnight at $4^{\circ} \mathrm{C}$ at a 1:100 dilution in $1 \times$ PBS containing $2 \%$ BSA. Overnight incubation was followed by 3 min $\times 10$ min washes in $1 \times$ PBS, followed by incubation in Alexa Fluor 488 secondary antibody for $2 \mathrm{~h}$ in the dark at room temperature. Slides were examined under a confocal laserscanning microscope (Zeiss LSM 510 META) with a Plan Apo Chromat X63 oil objective (N.A. 1.4), and immunofluorescent (IF) images were captured using LSM 510 META software version 3.2.

\section{Transwell Migration Assay}

Following 24 and $48 \mathrm{~h}$ of treatment with either $1 \mu \mathrm{M}$ calcitriol or control, cells were seeded into the upper chamber of a transwell insert pre-coated with $5 \mu \mathrm{g} / \mathrm{mL}$ fibronectin for migration in serum-free medium at a density of 300000 cells per well (24-well insert; $8 \mu \mathrm{m}$ pore size, BD Biosciences). Medium containing $10 \%$ fetal bovine serum (FBS) was placed in the lower chamber as a chemoattractant, and cells were further incubated for $24 \mathrm{~h}$. Nonmigratory cells were removed from the upper chamber by swabbing the upper surface of the transwell membrane with a cotton bud. The migratory cells remaining on the lower surface of the insert were fixed and stained using Diff-Quick dye (Dade Behring, Inc, B4132-1A). Cells were quantified as the average number of cells in five random highpowered fields in three independent inserts.

\section{Clonogenic Survival Assay}

For analysis of radiation therapy, cells were seeded in a 6-well plate and treated with a range of radiation from 0 to $6 \mathrm{~Gy}$ delivered using a Varian Clinac 21EX linear accelerator. Cells were cultured for $10 \mathrm{~d}$ until colonies formed. The colonies were then washed with $1 \times$ PBS and stained with a crystal violet dye. Total cell colonies were counted per well. Surviving colonies were defined as groups of 50 or more cells growing together. To assess the ability of calcitriol to sensitize cells to radiation, cells were plated in a 24 well plate, pretreated with calcitriol or vehicle control for $24 \mathrm{~h}$ prior to radiation treatment. Cells were cultured for $10 \mathrm{~d}$ and then washed, stained, and counted as described above.

\section{Statistical Analysis}

Statistical analyses were performed using the paired Student's $t$-test. $P<0.05$ was considered significant.

\section{RESULTS}

\section{Variable Expression of EMT Markers and Vitamin D Receptor in a Panel of Colon Cancer Cell Lines}

We surveyed a panel of colon cancer cell lines for markers of EMT including E-cadherin, vimentin, Snail and Slug (Figure 1). E-cadherin protein and mRNA expression as well as Snail and Slug mRNA levels demonstrated variable expression across the panel of cell lines. The well-characterized pair of cell lines SW480 (derived from a primary tumor) and SW620 (metastatic tumor) cell lines had the most mesenchymal features including high vimentin and low E-cadherin expression compared with other cell lines tested [23,24]. Furthermore, 
SW620 cells had the highest level of Slug expression and one of the highest levels of Snail expression. DLD1, HCT116, and HT29 had the least mesenchymal features including low/ undetectable vimentin and high E-cadherin expression. An inverse correlation was observed between Snail and Slug mRNA and E-cadherin expression levels in our panel of cell lines, and all cell lines tested expressed the VDR, suggesting that these cell lines may be a good model system to evaluate the potential of calcitriol modulation of EMT markers through the VDR.

\section{Calcitriol Treatment Variably Augments E-Cadherin Expression}

Although a reciprocal relationship may exist between EMT and VDR, the ability of calcitriol to augment expression of critical mediators of EMT remains uncertain. To explore the effect of calcitriol on E-cadherin and critical EMT regulators we chose three cell lines from our panel, DLD1, HCT116, and SW620, based on their variable VDR, E-cadherin, Slug and Snail expression. We treated with calcitriol for 24 and $48 \mathrm{~h}$ and assessed Ecadherin expression by Western Blot and immunofluorescence (IF) for localization (Figure 2). In the DLD-1 cells, total E-cadherin expression appeared stable over time with calcitriol treatment, however, IF staining demonstrated increased E-cadherin cell membrane localization after $48 \mathrm{~h}$. In the HCT116 cell line, increased E-cadherin protein expression was observed after $48 \mathrm{~h}$ by Western blot and IF staining. Conversely, in response to calcitriol, the SW620 cells demonstrated decreased E-cadherin expression after $48 \mathrm{~h}$ as demonstrated by Western blot and IF (Figure 2). These data suggest that calcitriol has differential effects on E-cadherin in a cell context manner, suggesting the mesenchymal nature of the cell might determine the beneficial effects of calcitriol.

\section{Calcitriol Treatment Augments Expression of Critical E-Cadherin Transcriptional Regulators}

We further evaluated the effect of calcitriol on the expression of E-cadherin transcription mediators, Snail and Slug by qRT-PCR (Figure 3). In the DLD-1 and HCT116 cell lines, both Slug and Snail mRNA expression levels were decreased after treatment with calcitriol $(P<0.05)$. Conversely, both Snail and Slug mRNA levels were significantly increased in the SW620 cells following calcitriol treatment by $48 \mathrm{~h}(P<0.05)$. A similar pattern was observed with vimentin in response to calcitriol treatment. In the DLD-1 and HCT116 cells, vimentin mRNA expression was decreased at 24 and $48 \mathrm{~h}(P<0.05)$. Conversely, the SW620 cells demonstrated significantly enhanced vimentin expression at $24 \mathrm{~h}$ that returned to baseline at $48 \mathrm{~h}(P<0.05)$. Taken together, these data suggest that calcitriol can augment EMT transcriptional regulators in a cell line dependent fashion. The finding that calcitriol can induce Snail, Slug and vimentin in the SW620 might explain the observed loss of Ecadherin expression at the protein level and suggests that calcitriol has the potential to enhance EMT at a molecular level in certain cells.

\section{Calcitriol Treatment Inhibits Colon Cancer Cell Migration}

With the ability of calcitriol to regulate expression of EMT mediators, we decided to assess the functional significance of calcitriol treatment on colon cancer cells. To investigate the ability of calcitriol to augment colon cancer cell migration, human colon cancer cells were pre-treated with calcitriol for 24 and $48 \mathrm{~h}$ prior to assessing migration with a Boyden chamber assay (Figure 4). In the DLD-1 and HCT116 cell lines that demonstrated decreased expression of Slug in response to calcitriol treatment, $48 \mathrm{~h}$ pre-treatment with calcitriol significantly suppressed cellular migration by $62 \%$ and $80 \%$, respectively $(P<0.02)$. In contrast, the SW620 cells with the lowest levels of E-cadherin and the highest levels of Slug expression demonstrated no significant change in migration with calcitriol pre-treatment. 


\section{Radiation Sensitizing Ability of Calcitriol Is Inversely Associated With EMT Features}

Besides the well-established role of EMT in cellular motility, EMT has also been associated with increased cellular survival and decrease apoptosis $[17,18]$. Therefore, we hypothesized that associated with inhibition of EMT, calcitriol treatment would enhance radiation cytotoxicity. To determine the effect of calcitriol on radiation sensitivity of colon cancer cells, cells were pre-treated with calcitriol for $24 \mathrm{~h}$ followed by radiation doses of 0-6 Gy (Figure 5). Based on the clonogenic assay, the panel of colon cancer cell lines demonstrated variable sensitivity to radiation (Figure 5). Pre-treatment with calcitriol alone led to variable colony survival across a panel of cell lines. In the DLD-1 and HCT116 cell lines that demonstrated reduced Snail and Slug in response to calcitriol treatment, $24 \mathrm{~h}$ calcitriol pretreatment enhanced the radiation sensitivity by 2.3 - and 2.6 -fold respectively compared with vehicle control at $4 \mathrm{~Gy}(P<0.05)$. Interestingly, the SW620 cell line showed no change in radiation sensitivity with calcitriol pre-treatment. The SW620 cells demonstrated increased Slug and Snail expression with calcitriol treatment (Figure 3), suggesting that Slug and/or Snail may inhibit calcitriol mediated sensitivity to radiation.

\section{Overexpression of Slug Inhibits the Radiation Sensitizing Ability of Calcitriol Pre- Treatment}

Snail and Slug, transcriptional mediators of EMT, can repress activity of the VDR promoter $[13,14,20]$. Based on our observation that mesenchymal features such as low E-cadherin, high vimentin and high Slug expression as demonstrated in the SW620 cells is associated with resistance to calcitriol radiation sensitizing effects, we further explored this finding by overexpressing Snail and Slug in two human colon cancer cell lines (DLD-1 and HCT 116 cells) that demonstrated enhanced radiation sensitivity with calcitriol pre-treatment (Figure 6A). In both the DLD-1 and HCT116 cell lines, Slug overexpression resulted in the greatest loss of E-cadherin relative to the empty vector control and Snail overexpression cell lines (Figure 6B). Furthermore, VDR expression was not decreased with either Snail or Slug overexpression. Compared with the empty vector controls, calcitriol was able to sensitize both Snail DLD-1 and Snail HCT116 cells to radiation (Figure 6C), similar to that observed in the parental cell lines (Figure 5). Indeed, the expression of Snail in the DLD-1 cells appeared to enhance the calcitriol -induced sensitivity to radiation treatment. However, both the Slug DLD-1 and Slug HCT116 cells demonstrated resistance to the sensitizing effects of calcitriol therapy (Figure 6C). Taken together, these data suggest that the EMT transcriptional factor Slug can inhibit calcitriol radiation sensitizing ability independent of a direct effect on the expression of VDR.

\section{DISCUSSION}

Calcitriol (the hormonally active form of Vitamin D) has demonstrated the ability to sensitize cancer cells in vitro to both chemotherapy and radiation and has previously shown promise in clinical trials in prostate and head and neck squamous cell carcinoma cancer clinical trials although treatment effect has been sporadic $[4,25,26]$. The use of calcitriol concurrently with traditional anti-neoplastic therapies is very attractive especially with the low toxicity profile of calcitriol even at high doses [27]. Currently, the use of calcitriol as a therapeutic agent in colon cancer remains unexplored and no molecular biomarkers have been utilized to select appropriate patients for calcitriol based therapy.

Our investigation demonstrates for the first time the ability of calcitriol to enhance the in vitro therapeutic effects of radiation in colon cancer cell lines and highlights Slug as a critical molecular mediator augmenting the therapeutic effects of calcitriol. Furthermore, our findings demonstrate the ability of calcitriol to enhance the epithelial phenotype/repress mesenchymal features in certain cancer cell lines. We defined cell lines as calcitriol 
sensitive based on demonstrating an enhanced epithelial phenotype with increased Ecadherin, reduced vimentin and decreased expression of Snail and Slug as well as decreased cellular migration in response to calcitriol. In calcitriol sensitive cells, calcitriol demonstrated enhanced radiation sensitivity by up to $38 \%$. In contrast, cells with high mesenchymal features including high Slug and vimentin expression with low E-cadherin expression (SW620) demonstrated no significant growth inhibition in response to calcitriol treatment. Similarly, transfection of Slug in the calcitriol sensitive colon cancer cell lines, DLD-1 and HCT 116, completely inhibited the radiation sensitizing effect of calcitriol further supporting the conclusion that expression of Slug mitigates the anti-neoplastic effects observed with calcitriol pre-treatment. Interestingly, Snail overexpression in the sensitive cell lines did not suppress the therapeutic benefit of calcitriol. Importantly, calcitriol did not decrease response to radio-therapy in any cell line including the SW620 cells that demonstrated induced Slug expression following calcitriol treatment. Dose levels evaluated in our experiments are high $(1 \mu \mathrm{M})$ as lower dose did not enhance radiation sensitivity in our colon cancer cell lines. Collectively, we demonstrate that calcitriol can enhance the therapeutic effects of radiation in colon cancer cells and Slug expression mitigates this observed effect potentially representing an effective biomarker for calcitriol therapy.

The reciprocal relationship between calcitriol and EMT has been previously reported $[13,14,20]$. Calcitriol treatment can induce E-cadherin, a critical mediator of EMT, in cancer cells as we also demonstrated. On the other hand, Snail and Slug, transcriptional EMT mediators, can repress the VDR gene promoter alone or in an additive fashion and can block the induction of E-cadherin in response to calcitriol $[13,14,20]$. Our investigations further support this reciprocal relationship in cells sensitive to calcitriol. In calcitriol sensitive cell lines, we demonstrated decreased migration as well as induction of E-cadherin expression in response to calcitriol. The calcitriol sensitive cell lines also demonstrated enhanced radiation cytotoxicity with calcitriol pretreatment. In contrast, SW620 cell demonstrating a mesenchymal phenotype with high baseline Slug, high vimentin expression and low Ecadherin were resistant to the anti-neoplastic effects of calcitriol. This ability of Slug to negate the radiation sensitizing effects of calcitriol was further demonstrated in the DLD-1 and HCT116 cell lines when Slug was overexpressed. Furthermore, calcitriol treatment of SW620 cells induced expression of EMT molecular changes enhancing expression of Slug and repressing E-cadherin expression over $48 \mathrm{~h}$. A similar observation was previously reported highlighting the ability of calcitriol and calcitriol analogues to stimulate proneoplastic signaling in cells with high osteopontin and low E-cadherin supporting our conclusions that relative expression of critical VDR downstream genes may predict the response to calcitriol treatment (Figure 7) [28]. The ability of calcitriol to stimulate critical mediators of EMT such as Slug under certain conditions warrants concern as it implies a proneoplastic effect in select cancer cells and requires further investigation. Given the metastatic characteristics of the SW620 colon cancer cell line, our data suggests that with advanced (metastatic) cancers the biology of calcitriol signaling may be altered to enhance neoplastic progression. Further investigations may be important prior to clinical trials incorporating calcitriol for advanced malignancies. Although not explored in this study, the presence of VDR polymorphisms in the various colon cancer cell lines may be another explanation for the reciprocal effect we observed with calcitriol treatment and warrants future investigation [29].

To date, the therapeutic benefit of calcitriol has been inconsistent in clinical trials. Our investigations suggest that Slug expression may inhibit the benefit of adjuvant calcitriol in colon cancer and along with low E-cadherin expression could represent a calcitriol therapeutic biomarker. 
In conclusion, calcitriol enhanced radiation sensitivity in colon cancer cell lines with the least mesenchymal features and expression of Slug conferred resistance to this effect. Novel calcitriol therapeutic strategies appear promising as radiation sensitizers but may need to be individualized based on expression of EMT mediators.

\section{Acknowledgments}

Grant sponsor: SCTR NIH/NCRR; Grant number: UL1RR029882; Grant sponsor: NIH; Grant number: 1K08CA142904

This publication was supported by the South Carolina Clinical \& Translational Research Institute with an academic home at the Medical University of South Carolina CTSA, NIH/NCRR grant number UL1RR029882. The contents are solely the responsibility of the authors and do not necessarily represent the official views of the NIH or NCRR.

\section{Abbreviations}

calcitriol

EMT

VDR

empty HCT116

empty DLD-1

qRT-PCR

GAPDH

IF 1a,25-dihydroxyvitamin $\mathrm{D}_{3}$

epithelial-to-mesenchymal transition

vitamin-D receptor

HCT116 colon cancer cells overexpressing Slug (Slug HCT116), Snail (Snail HCT116) or empty

DLD-1 colon cancer cells overexpressing Slug (Slug DLD-1), Snail (Snail DLD-1) or empty

reverse transcription-PCR

glyceraldehyde-3-phosphate dehydrogenase

immunofluorescence

\section{References}

1. Sauer R, Becker H, Hohenberger W, et al. Preoperative versus postoperative chemoradiotherapy for rectal cancer. N Engl J Med. 2004; 351:1731-1740. [PubMed: 15496622]

2. Guillem JG, Chessin DB, Cohen AM, et al. Long ${ }^{\mathrm{Q} 3}$-term oncologic outcome following preoperative combined modality therapy, total mesorectal excision of locally advanced rectal cancer. Ann Surg. 2005; 241:829-836. discussion 836-848. [PubMed: 15849519]

3. Deeb KK, Trump DL, Johnson CS. Vitamin D signalling pathways in cancer potential for anticancer therapeutics. Nat Rev Cancer. 2007; 7:684-700. [PubMed: 17721433]

4. Ma Y, Trump DL, Johnson CS. Vitamin D in combination cancer treatment. J Cancer. 2010; 1:101107. [PubMed: 20842231]

5. Yu WD, Ma Y, Flynn G, et al. Calcitriol enhances gemcitabine anti-tumor activity in vitro, in vivo by promoting apoptosis in a human pancreatic carcinoma model system. Cell cycle. 2010; 9:30223029. [PubMed: 20699664]

6. Ahmed S, Johnson CS, Rueger RM, Trump DL. Calcitriol (1,25-dihydroxycholecalciferol), potentiates of activity mitoxantrone/dexamethasone in an androgen independent prostate cancer model. J Urol. 2002; 168:756-761. [PubMed: 12131364]

7. Hershberger PA, Yu WD, Modzelewski RA, et al. Calcitriol (1,25-dihydroxycholecalciferol) enhances paclitaxel antitumor activity in vitro, in vivo, accelerates paclitaxel-induced apoptosis. Clin Cancer Res. 2001; 7:1043-1051. [PubMed: 11309356]

8. Xu Y, Fang F, St Clair DK, et al. Suppression of RelB-mediated manganese superoxide dismutase expression reveals a primary mechanism for radiosensitization effect of 1alpha, 25dihydroxyvitamin D(3) in prostate cancer cells. Mol Cancer Ther. 2007; 6:2048-2056. [PubMed: 17604335] 
9. Kane KF, Langman MJ, Williams GR. Antiproliferative responses to two human colon cancer cell lines to vitamin D3 are differently modified by 9-cis-retinoic acid. Cancer Res. 1996; 56:623-632. [PubMed: 8564982]

10. Lointier P, Wargovich MJ, Saez S, et al. The role of vitamin D3 in the proliferation of a human colon cancer cell line in vitro. Anticancer Res. 1987; 7:817-821. [PubMed: 2823686]

11. Palmer HG, Sanchez-Carbayo M, Ordonez-Moran P, et al. Genetic signatures of differentiation induced by 1alpha,25-dihydroxyvitamin D3 in human colon cancer cells. Cancer Res. 2003; 63:7799-7806. [PubMed: 14633706]

12. Palmer HG, Gonzalez-Sancho JM, Espada J, et al. Vitamin D(3) promotes the differentiation of colon carcinoma cells by the induction of E-cadherin, the inhibition of beta-catenin signaling. $\mathrm{J}$ Cell Biol. 2001; 154:369-387. [PubMed: 11470825]

13. Palmer HG, Larriba MJ, Garcia JM, et al. The transcription factor SNAIL represses vitamin D receptor expression, responsiveness in human colon cancer. Nat Med. 2004; 10:917-919. [PubMed: 15322538]

14. Larriba MJ, Martin-Villar E, Garcia JM, et al. Snail2 cooperates with Snail1 in the repression of vitamin D receptor in colon cancer. Carcinogenesis. 2009; 30:1459-1468. [PubMed: 19502595]

15. Batlle E, Sancho E, Franci C, et al. The transcription factor snail is a repressor of E-cadherin gene expression in epithelial tumour cells. Nat Cell Biol. 2000; 2:84-89. [PubMed: 10655587]

16. Cano A, Perez-Moreno MA, Rodrigo I, et al. The transcription factor snail controls epithelialmesenchymal transitions by repressing E-cadherin expression. Nat Cell Biol. 2000; 2:76-83. [PubMed: 10655586]

17. Vega S, Morales AV, Ocana OH, et al. Snail blocks the cell cycle, confers resistance to cell death. Genes Dev. 2004; 18:1131-1143. [PubMed: 15155580]

18. Kajita M, McClinic KN, Wade PA. Aberrant expression of the transcription factors snail slug alters the response to genotoxic stress. Mol Cell Biol. 2004; 24:7559-7566. [PubMed: 15314165]

19. Yang AD, Fan F, Camp ER, et al. Chronic oxaliplatin resistance induces epithelial-tomesenchymal transition in colorectal cancer cell lines. Clin Cancer Res. 2006; 12:4147-4153. [PubMed: 16857785]

20. Larriba MJ, Bonilla F, Munoz A. The transcription factors Snail1 Snail2 repress vitamin D receptor during colon cancer progression. J Ster Biochem Mol Biol. 2010; 121:106-109.

21. Camp ER, Findlay VJ, Vaena SG, et al. Slug Q4 expression enhances tumor formation in a noninvasive rectal cancer model. J Surg Res. 2011

22. Camp ER, Findlay VJ, Vaena SG, et al. Slug expression enhances tumor formation in a noninvasive rectal cancer model. J Surg Res. 2011; 170:56-63. [PubMed: 21470622]

23. Gagos S, Hopwood VL, Iliopoulos D, et al. Chromosomal markers associated with metastasis in two colon cancer cell lines established from the same patient. Anticancer Res. 1995; 15:369-378. [PubMed: 7763008]

24. Maehle L, Eilertsen E, Mollerup S, et al. Effects of n-3 fatty acids during neoplastic progression, comparison of in vitro, in vivo sensitivity of two human tumour cell lines. Br J Cancer. 1995; 71:691-696. [PubMed: 7710930]

25. Flaig TW, Barqawi A, Miller G, et al. A phase II trial of dexamethasone, vitamin D, carboplatin in patients with hormone-refractory prostate cancer. Cancer. 2006; 107:266-274. [PubMed: 16779800]

26. Walsh JE, Clark AM, Day TA, et al. Use of alpha, 25-dihydroxyvitamin D3 treatment to stimulate immune infiltration into head, neck squamous cell carcinoma. Hum Immunol. 2010; 71:659-665. [PubMed: 20438786]

27. Chadha MK, Tian L, Mashtare T, et al. Phase 2 trial of weekly intravenous 1,25 dihydroxy cholecalciferol (calcitriol) in combination with dexamethasone for castration-resistant prostate cancer. Cancer. 2010; 116:2132-2139. [PubMed: 20166215]

28. Xu H, McCann M, Zhang Z, et al. Vitamin D receptor modulates the neoplastic phenotype through antagonistic growth regulatory signals. Mol Carcinogen. 2009; 48:758-772.

29. Jacobs ET, Van Pelt C, Forster RE, et al. CYP24A1 Q5, CYP27B1 polymorphisms modulate vitamin D metabolism in colon cancer cells. Cancer Res. 2013 
A
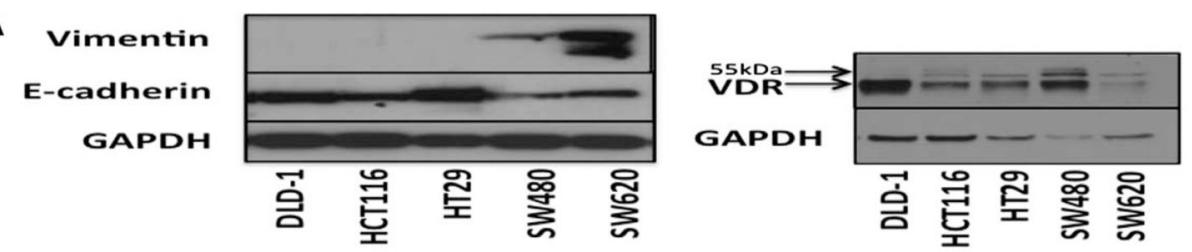

B
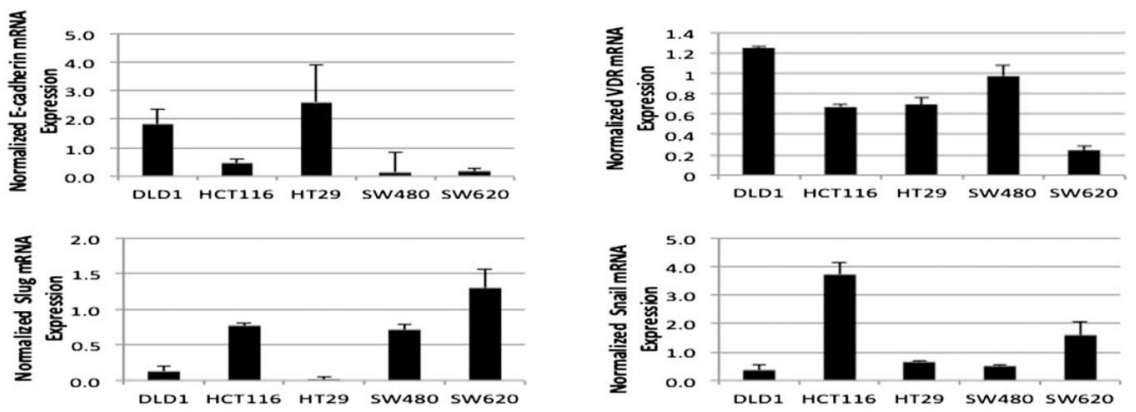

Figure 1.

Variable expression of EMT markers and Vitamin D receptor in a panel of colon cancer cell lines. (A) Western Blot analysis of vimentin, E-cadherin, and VDR from total cell lysates of various colon cancer cell lines. GAPDH was used as a loading control. (B) Real-time RTPCR analysis of E-cadherin, VDR, Slug, and Snail mRNA expression normalized to GAPDH. 

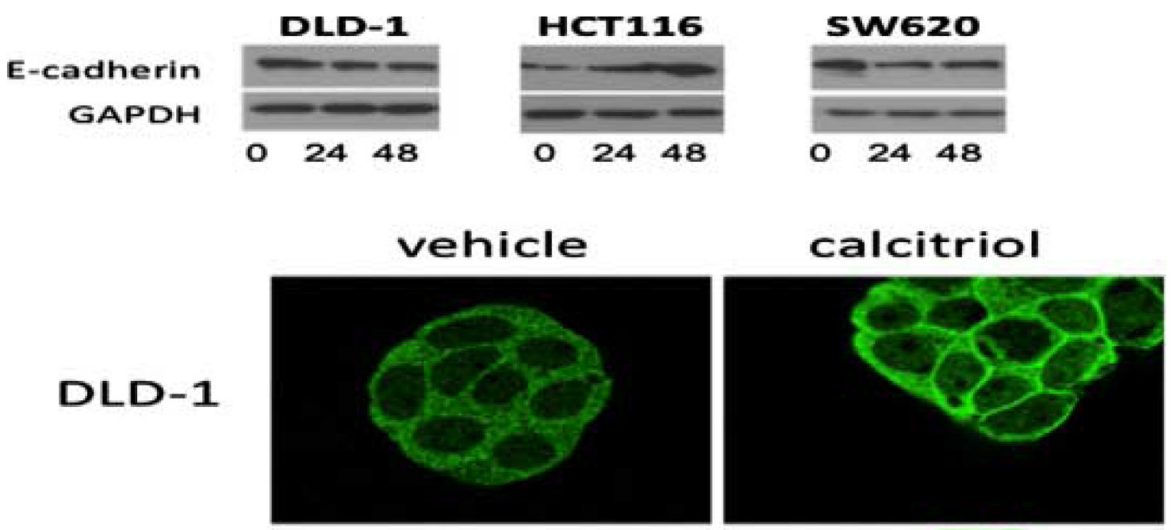

HCT116
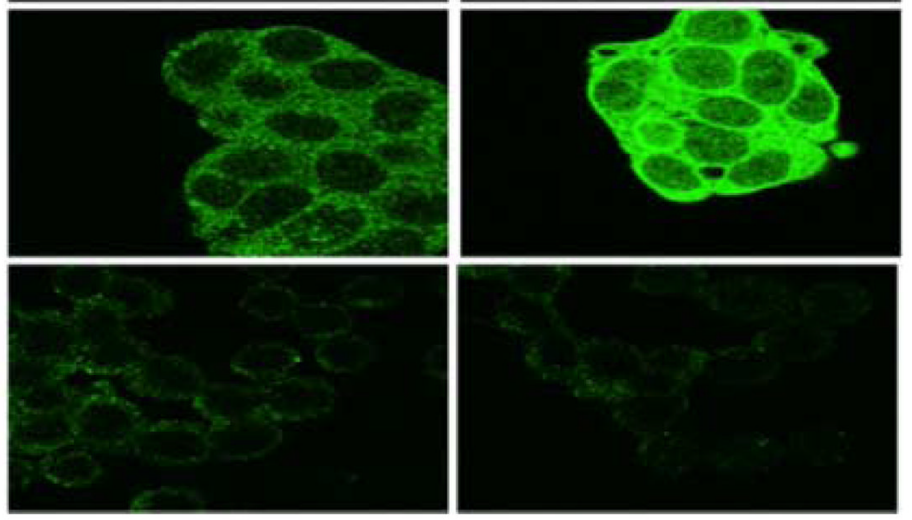

Figure 2.

Calcitriol treatment augments expression of E-cadherin in colon cancer cells. (A) Western Blot analysis of E-cadherin in DLD1, HCT116, and SW620 colon cancer cells treated with 1 $\mu \mathrm{M}$ calcitriol for the indicated time in hours. GAPDH was used as a loading control. (B) Immunofluorescent E-cadherin staining after $48 \mathrm{~h}$ of $1 \mu \mathrm{M}$ calcitriol (right panels) or vehicle control (left panels) treatment. 
A

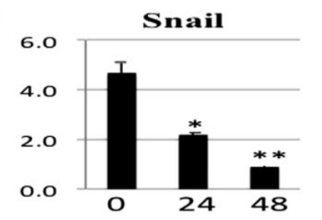

B

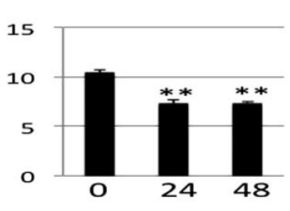

C

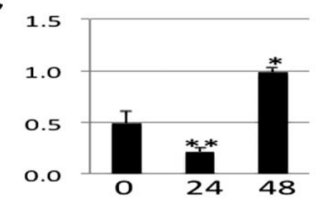

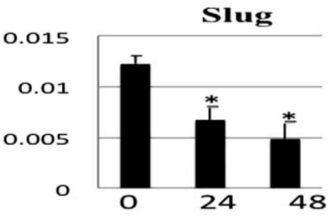
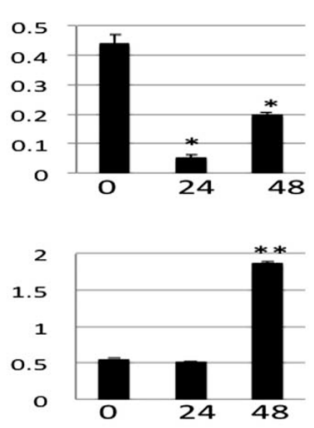
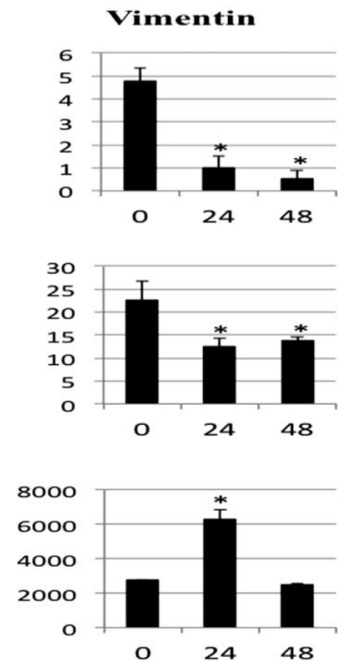

Figure 3.

Calcitriol treatment alters expression of Snail and Slug. Real-time RT-PCR analysis of Snail (left panels), Slug (middle panels), and Vimentin (right panels) from (A) DLD-1, (B) HCT116, and (C) SW620 colon cancer cells treated with $1 \mu \mathrm{M}$ calcitriol for the indicated time in hours. $* P<0.05, * * P<0.005$. 

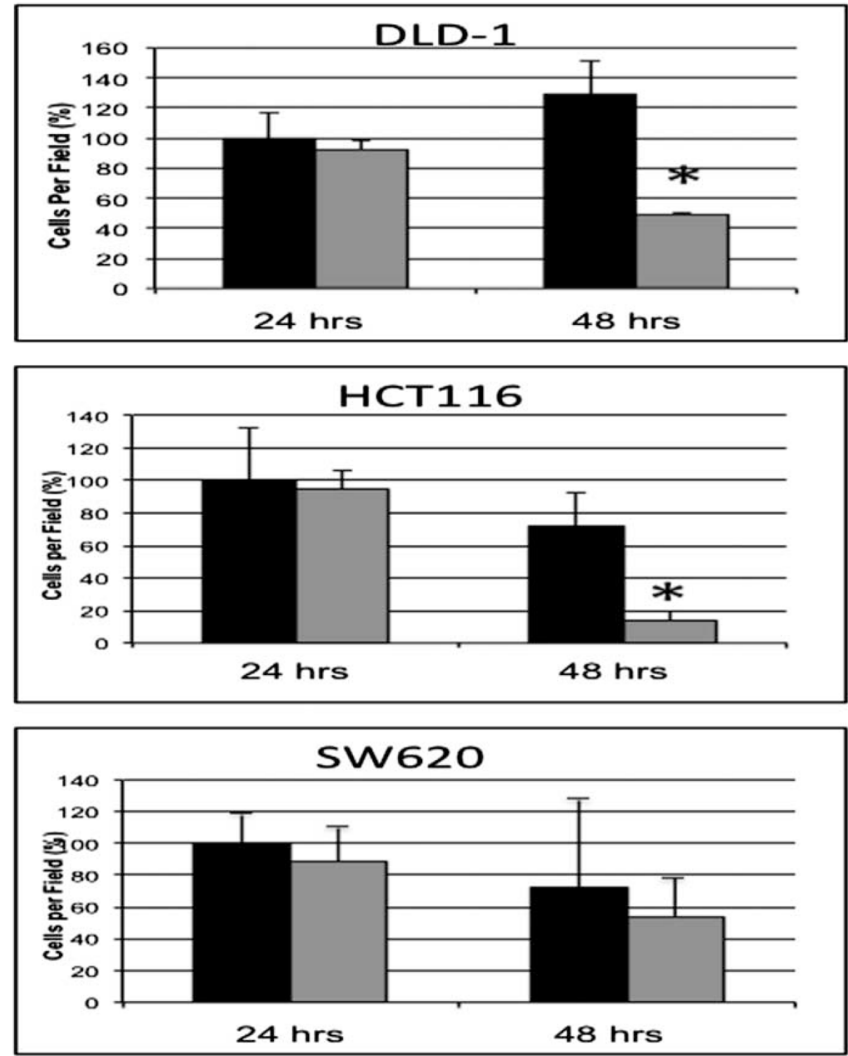

Figure 4.

Calcitriol pre-treatment variably regulates colon cancer cellular migration. DLD-1, HCT116, and SW620 colon cancer cells were pre-treated with $1 \mu \mathrm{M}$ calcitriol (gray bars) or vehicle alone (black bars) for 24 and $48 \mathrm{~h}$, then plated on Boyden migration chamber inserts. Cellular migration was quantified as mean number of cells migrating through the transwell inserts. Columns, average $\%$ of cells invading per high-powered field. $* P<0.02$. 


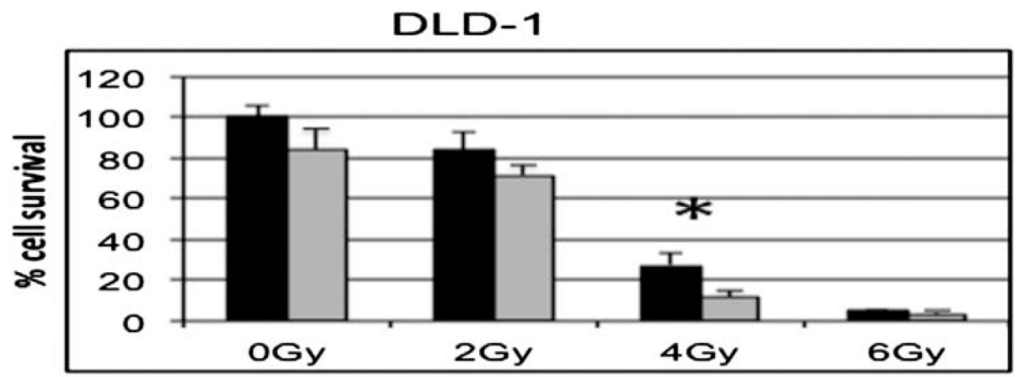

HCT 116

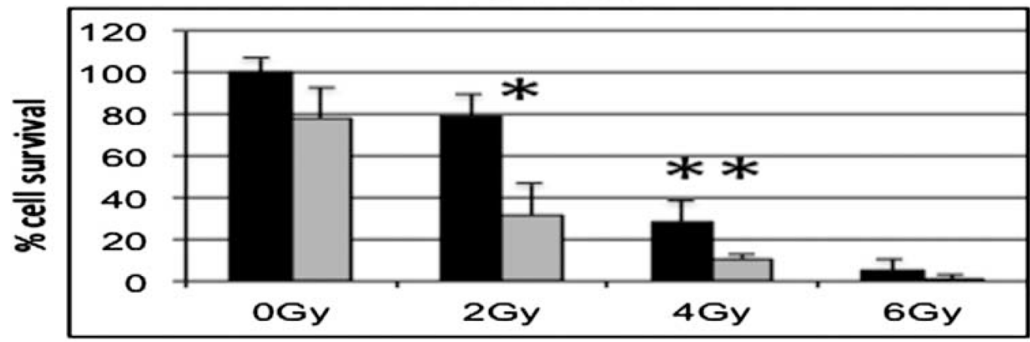

SW620

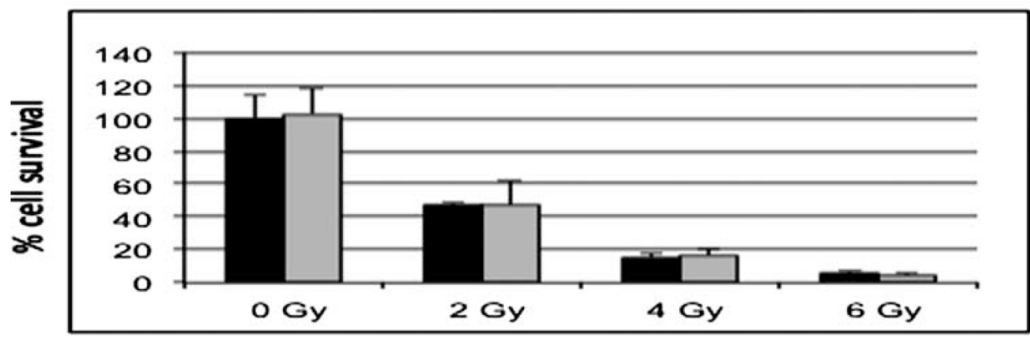

Figure 5.

Calcitriol sensitizes colon cancer cells to radiation. DLD-1, HCT116, and SW620 colon cancer cells were pre-treated with $1 \mu \mathrm{M}$ calcitriol (gray bars) or vehicle (black bars) for $24 \mathrm{~h}$ prior to radiation treatment of $0-6 \mathrm{~Gy}$. After $10 \mathrm{~d}$, surviving colonies of 20 or more cells were counted to determine the $\%$ survival relative to the untreated cells. $* P<0.01$, $* * P<$ 0.05 . 
A

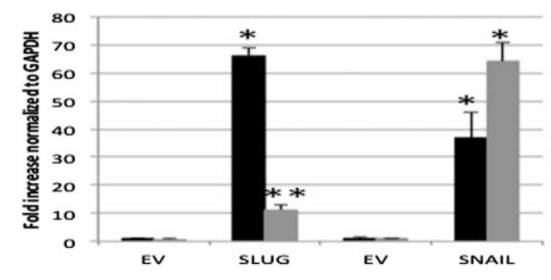

C

Empty

(2)

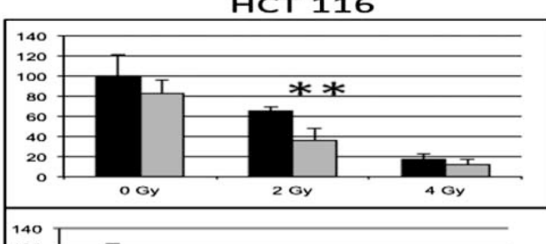

Snail

20

slug
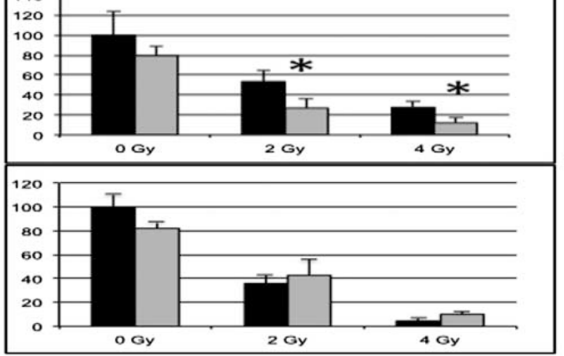

B

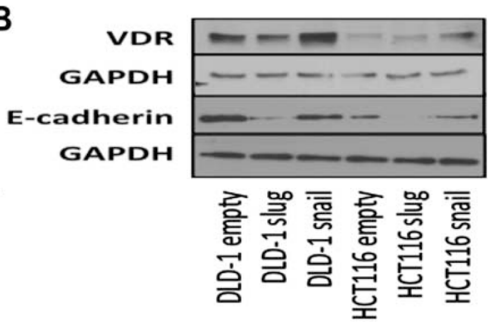

DLD-1

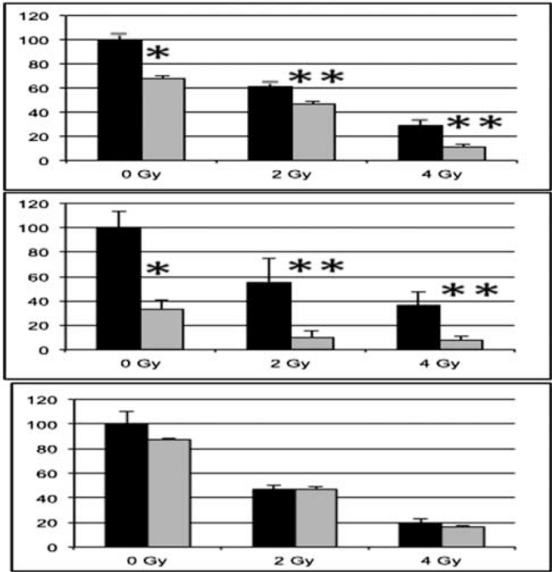

Figure 6.

Overexpression of Slug inhibits calcitriol's radiation sensitizing ability. (A) Real-time RTPCR confirmed expression of Snail and Slug in stable transfected pools of both Snail and Slug overexpressing DLD-1 (black bars) and HCT116 (gray bars) cells compared to the empty vector (EV) controls. Data were normalized to GAPDH. (B) Western Blot analysis of VDR and E-cadherin from total cell lysates of Snail and Slug expressing HCT116 and DLD-1 cells compared to the empty vector controls. GAPDH was used as a loading control. (C) Cell lines were pre-treated with $1 \mu \mathrm{M}$ calcitriol (gray bars) or vehicle alone (black bars) for $24 \mathrm{~h}$ prior to radiation treatment of $0-6 \mathrm{~Gy}$ and compared with empty vector stably transfected cells. After $10 \mathrm{~d}$, surviving colonies of 20 or more cells were counted to determine the $\%$ survival relative to the untreated cells. $* P<0.01, * * P<0.05$. 


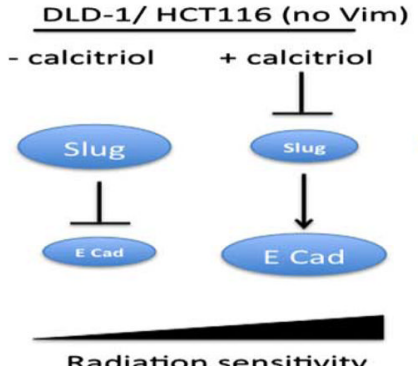

Radiation sensitivity

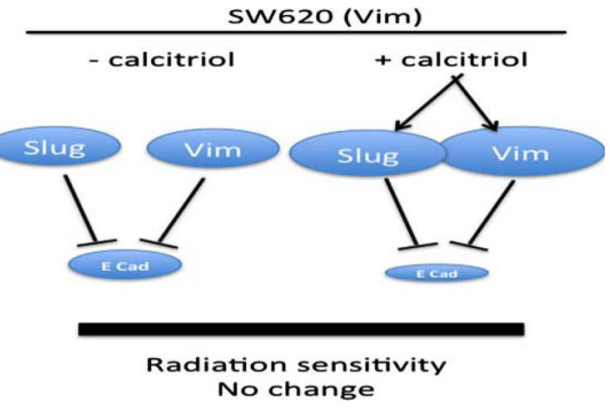

Figure 7.

Model of calcitriol mediated sensitivity to radiation. In DLD-1 and HCT116 cells, Slug is a negative regulator of E-cadherin. The addition of calcitriol leads to repression of Slug and a subsequent increase in E-cadherin (E Cad) and/or membrane localized E-cadherin resulting in an increased sensitivity to radiation. However, in SW620 cells in which both Slug and vimentin (Vim) are induced with the addition of calcitriol, calcitriol therapy does not increase E-cadherin levels and therefore we see no change in radiation sensitivity. 